\title{
Análise da Revisão Cochrane: Terapêutica Farmacológica da Hiperuricemia em Doentes Hipertensos. Cochrane Database Syst Rev. 2017;4:CD008652.
}

\author{
Analysis of the Cochrane Review: Pharmacotherapy for \\ Hyperuricemia in Hypertensive Patients. Cochrane \\ Database Syst Rev. 2017;4:CD008652.
}

\author{
Miguel BIGOTTE VIEIRA ${ }^{* 1}$, Rute Baeta BAPTISTA*2,3, João COSTA ${ }^{4,5}$, António VAZ-CARNEIRO $\ 4,5$
}

Acta Med Port 2017 May;30(5):356-360 - https://doi.org/10.20344/amp.9173

\section{RESUMO}

A hipertensão arterial é um problema de saúde pública que afeta cerca de $25 \%$ da população adulta mundial. A associação entre hiperuricemia e hipertensão arterial tem sido demonstrada em estudos epidemiológicos e experimentais. No entanto, não é claro se a terapêutica hipouricemiante reduz os valores de pressão arterial. Esta revisão sistemática - uma versão atualizada de outra previamente publicada - teve como objetivo primário avaliar o efeito da terapêutica hipouricemiante nos valores de pressão arterial de doentes com pré-hipertensão ou hipertensão arterial primárias. Os objetivos secundários foram avaliar a eficácia da terapêutica na redução da uricemia e o perfil de segurança. Foram selecionados ensaios clínicos aleatorizados ou quasi-aleatorizados que comparassem o efeito nos valores de pressão arterial da terapêutica hipouricemiante versus placebo, em doentes com hiperuricemia e pré-hipertensão ou hipertensão arterial essencial. Foram pesquisadas as seguintes bases de dados até fevereiro de 2016: The Cochrane Hypertension Specialised Register, the Cochrane Central Register of Controlled Trials (CENTRAL), Medline, Embase, The World Health Organization International Clinical Trials Registry Platform, e ClinicalTrials.gov. Foi também pesquisada a LILACS até março de 2016 e contactados os autores dos estudos considerados relevantes. Dos 349 artigos identificados, foram pré-selecionados 21, tendo sido incluídos três ensaios clínicos aleatorizados (211 doentes) na análise qualitativa e na meta-análise. Dois destes ensaios incluíram exclusivamente adolescentes. Os autores concluem que a terapêutica hipouricemiante não é eficaz na redução da pressão arterial na população de doentes com hiperuricemia e pré-hipertensão ou hipertensão arterial essencial. No entanto, esta estratégia poderá ser mais eficaz na população específica dos adolescentes com pré-hipertensão ou hipertensão arterial ligeira diagnosticada recentemente. A terapêutica hipouricemiante é eficaz na redução do valor sérico de ácido úrico e a suspensão da terapêutica devido a efeitos adversos não foi superior nos grupos tratados comparativamente com placebo (embora um doente a tenha suspendido por reação cutânea grave).

Palavras-chave: Alopurinol; Hipertensão; Hiperuricemia; Pressão Sanguínea; Revisão Sistemática; Uricosúricos/uso terapêutico

\section{ABSTRACT}

Arterial hypertension is a public health problem that affects approximately $25 \%$ of the world's adult population. The association between hypertension and hyperuricemia has been shown on epidemiological and experimental studies. However, it is unclear whether lowering serum uric acid might lower blood pressure. This Cochrane systematic review - a revised edition of a previously published one intended as primary objective to evaluate the effect of hypouricemic drugs in patients with primary hypertension or prehypertension. The secondary objectives were to evaluate the efficacy and safety of hypouricemic drugs. A systematic search until February 2016 on controlled, randomized or quasi-randomized trials comparing the effect of hypouricemic drug versus placebo in hypertensive or prehypertensive patients was performed on the following databases: The Cochrane Hypertension Specialised Register, the Cochrane Central Register of Controlled Trials (CENTRAL), Medline, Embase, The World Health Organization International Clinical Trials Registry Platform, e ClinicalTrials.gov. LILACS database up to March 2016 was also searched and the authors of relevant studies were contacted. There were 349 identified papers, 21 were preselected and three randomized clinical trials (211 patients) were included in the qualitative analysis and in the meta-analysis. Two of the trials were conducted exclusively on adolescents. The authors conclude that hypouricemic drugs are not effective in lowering blood pressure in patients with hyperuricemia and primary prehypertension or hypertension. However, this strategy might be more effective in the specific population of adolescents with prehypertension or mild primary hypertension recently diagnosed. Hypouricemic drugs effectively reduce serum uric acid level and withdrawals of therapy due to adverse effects were not superior in the treated group, comparing to placebo; however, one patient withdrew due to a severe cutaneous reaction.

Keywords: Allopurinol; Blood Pressure; Hypertension; Hyperuricemia; Randomized Controlled Trials as Topic; Uricosuric Agents/ therapeutic use

\section{QUESTÃO CLÍNICA}

Será que os fármacos hipouricemiantes reduzem a pressão arterial (PA) em adolescentes e adultos com hiperuricemia e pré-hipertensão / hipertensão arterial primária?

\section{OBJETIVOS}

Objetivo primário:

1. Avaliar o efeito da terapêutica hipouricemiante na $\mathrm{PA}$, aferido pela variação da PA sistólica e diastólica

* Co-primeiros autores.

1. Serviço de Nefrologia e Transplantação Renal. Centro Hospitalar Lisboa Norte. Lisboa. Portugal.

2. Pediatria Médica. Área da Mulher, Criança e Adolescente. Hospital de Dona Estefânia. Centro Hospitalar de Lisboa Central. Lisboa. Portugal.

3. Departamento de Fisiopatologia. Faculdade de Medicina. Universidade de Lisboa. Lisboa. Portugal.

4. Centro de Estudos de Medicina Baseada na Evidência. Faculdade de Medicina. Universidade de Lisboa. Lisboa. Portugal.

5. Centro Colaborador Português da Rede Cochrane Iberoamericana. Lisboa. Portugal.

$\triangle$ Autor correspondente: António Vaz-Carneiro. avc@medicina.ulisboa.pt

Recebido: 08 de maio de 2017 - Aceite: 08 de maio de 2017 | Copyright @ Ordem dos Médicos 2017 
medidas em consulta ou em ambulatório durante 24 horas.

\section{Objetivos secundários:}

1. Avaliar a eficácia da terapêutica hipouricemiante, aferida pela variação do valor sérico de ácido úrico.

2. Avaliar o perfil de segurança da terapêutica hipouricemiante, aferido pela ocorrência de efeitos adversos (mortalidade, efeitos adversos graves não fatais, eventos cardiovasculares e suspensão da terapêutica devido a efeitos adversos).

\section{METODOLOGIA}

Revisão sistemática em que se selecionaram para análise ensaios clínicos aleatorizados ou quasi-aleatorizados (abertos, com ocultação simples ou com ocultação dupla), comparando a terapêutica hipouricemiante com placebo, em doentes com hiperuricemia e pré-hipertensão/hipertensão arterial primárias, de qualquer género, idade ou etnia. Foram incluídos ensaios paralelos e cruzados (com período mínimo de interrupção entre intervenções de duas semanas). Foram excluídos ensaios aleatorizados por clusters.

As intervenções incluíram a utilização de duas classes de agentes hipouricemiantes: inibidores da xantina-oxidase (alopurinol e febuxostate) e agentes uricosúricos (benzbromarona, benziodarona, probenecide, sulfinpirazona, ethebencid e zoxazolamina). $O$ agente uricosúrico ticrinafeno foi excluído pelo seu efeito direto conhecido na PA. A duração mínima da intervenção com terapêutica hipouricemiante foi de quatro semanas. A comparação foi feita com placebo.

Hipertensão arterial foi definida como PA sistólica $\geq 140$ $\mathrm{mmHg}$ e/ou PA diastólica $\geq 90 \mathrm{mmHg}$. Pré-hipertensão foi definida como PA sistólica entre 120 e 139 mmHg ou PA diastólica entre 80 e $89 \mathrm{mmHg}$, em adultos; e como PA $\geq$ $120 / 80 \mathrm{mmHg}$ ou PA sistólica ou diastólica médias $\geq$ percentil 90 e < percentil 95, em crianças. Hiperuricemia foi definida como ácido úrico sérico $>6 \mathrm{mg} / \mathrm{dL}$ em mulheres, > $7 \mathrm{mg} / \mathrm{dL}$ em homens e $>5,5 \mathrm{mg} / \mathrm{dL}$ em crianças e adolescentes.

Os resultados incluíram, como variáveis contínuas, a PA (medição em consulta e medição ambulatória durante 24 horas) e o valor sérico de ácido úrico. Estes foram avaliados como diferenças de médias com intervalo de confiança (IC) de 95\%. A ocorrência de suspensão da terapêutica devido a efeitos adversos, como variável dicotómica, foi aferida através do risco relativo com IC de 95\%.

Para identificar os ensaios clínicos relevantes foram pesquisadas as seguintes bases de dados até fevereiro de 2016: The Cochrane Hypertension Specialised Register, the Cochrane Central Register of Controlled Trials (CENTRAL), Medline, Embase, The World Health Organization International Clinical Trials Registry Platform, e ClinicalTrials.gov. Foi também pesquisada a LILACS até março de 2016 e contactados os autores dos estudos considerados relevantes.
Dois investigadores selecionaram e avaliaram independentemente a qualidade metodológica dos estudos (risco de vieses pelo instrumento Cochrane Collaboration' Risk of Bias, indicadores de eficácia, unidade de análise, dados em falta, graus de heterogeneidade e vieses de publicação).

A qualidade global da evidência para cada outcome foi avaliada através da abordagem proposta pelo Grades of Recommendation, Assessment, Development and Evaluation Working Group (GRADE Working Group).

\section{RESULTADOS}

Foram identificados 349 artigos com base na estratégia de pesquisa. Destes, três corresponderam a estudos a decorrer cujos resultados não se encontravam ainda disponíveis. Foram selecionados numa primeira fase 21 artigos para leitura integral. Destes, 18 foram excluídos (nove por ausência de controlo com placebo, quatro por inclusão de doentes com valor sérico de ácido úrico normal, dois por análise retrospetiva, dois por inclusão de doentes normotensos, um por problemas metodológicos). Por fim, foram incluídos três ensaios clínicos aleatorizados (211 doentes) na análise qualitativa e na meta-análise que cumpriam os critérios de inclusão - em dois destes ensaios a população era constituída por adolescentes (entre 11 e 17 anos de idade) com hiperuricemia e pré-hipertensão ou hipertensão arterial primária estádio 1 .

Os principais resultados foram:

- Existe evidência de baixa qualidade, com base nos resultados de três ensaios clínicos, que a terapêutica hipouricemiante não reduz a PA sistólica $(-6,2 \mathrm{mmHg}$, $\mathrm{IC}_{95 \%}-12,8$ a 0,5$)$ ou a PA diastólica $\left(-3,9 \mathrm{mmHg}, \mathrm{IC}_{95 \%}\right.$ -9,2 a 1,4) avaliadas por medição ambulatória da PA durante 24 horas;

- Existe evidência de baixa qualidade, com base nos resultados de dois ensaios clínicos, que a terapêutica hipouricemiante reduz a PA sistólica $\left(-8,4 \mathrm{mmHg}, \mathrm{IC}_{95 \%}\right.$ $-15,2$ a -1,6) mas não a PA diastólica $\left(-6,5\right.$ mmHg, $I_{95 \%}$ -13,6 a 0,7) avaliadas por medição em consulta;

- Existe evidência de elevada qualidade, com base nos resultados de três ensaios clínicos, que a terapêutica hipouricemiante reduz o valor sérico de ácido úrico $(-3,1$ $\mathrm{mg} / \mathrm{dL}, \mathrm{IC}_{95 \%}-3,8$ a $\left.-2,4\right)$;

- Existe evidência de muito baixa qualidade, com base nos resultados de três ensaios clínicos, que a suspensão da terapêutica devido a efeitos adversos não é superior com a terapêtica hipouricemiante, em comparação com placebo (risco relativo $=1,86, I_{95 \%} 0,43$ a 8,10$)$. No entanto, num dos ensaios, um doente medicado com alopurinol suspendeu terapêtica devido a uma reação cutânea grave.

$\mathrm{Na}$ Tabela 1 são apresentadas os principais resultados para as comparações previamente referidas, assim como a qualidade global da evidência para cada outcome. 


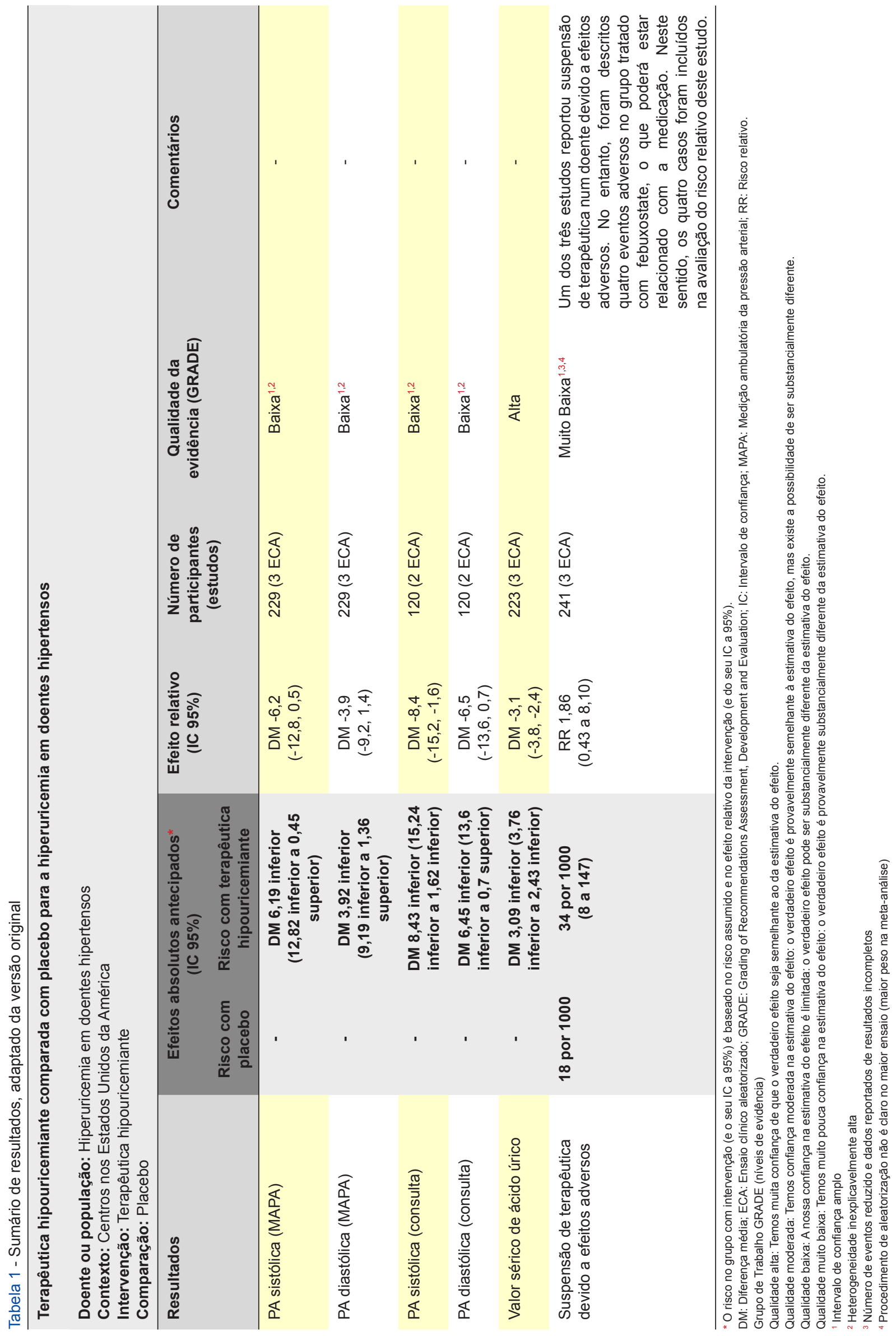




\section{CONCLUSÃO}

À data de realização da revisão sistemática, a evidência disponível baseada em ensaios clínicos aleatorizados é insuficiente para concluir se a terapêutica hipouricemiante é eficaz na redução da PA, sendo necessários mais estudos para responder a esta questão. A terapêutica hipouricemiante reduz eficazmente o valor sérico de ácido úrico. A evidência em relação à segurança é de muito baixa qualidade.

\section{COMENTÁRIO}

A hipertensão arterial é um problema de saúde pública que afeta cerca de $25 \%$ da população adulta mundial. A prevalência de hiperuricemia em indivíduos hipertensos é significativamente superior à encontrada na população geral e situa-se entre $25 \%$ a $40 \%$. A associação entre hiperuricemia e hipertensão arterial, conhecida desde o século XIX, foi demonstrada em inúmeros estudos epidemiológicos e experimentais. ${ }^{1}$ Alguns autores consideram a hiperuricemia como fator de risco cardiovascular. Discute-se, no entanto, a existência de uma relação de causalidade (versus mera associação) entre hiperuricemia, hipertensão arterial, doença cardiovascular e síndrome metabólico. ${ }^{2}$ Atualmente, não é claro se a terapêutica hipouricemiante tem algum impacto na redução dos valores de pressão arterial.

Os autores da revisão sistemática Cochrane pretenderam avaliar o efeito da terapêutica hipouricemiante nos valores de pressão arterial de doentes hiperuricémicos com pré-hipertensão ou hipertensão arterial primárias. A identificação de um eventual benefício poderia alargar a indicação para a utilização de agentes hipouricemiantes a doentes com hiperuricemia e pré-hipertensão/hipertensão arterial primária, independentemente da presença de gota.

Os resultados globais não permitem concluir que a terapêutica hipouricemiante seja eficaz na redução dos valores de PA em doentes com hiperuricemia e pré-hipertensão/ hipertensão arterial primária. No entanto, verificou-se uma redução significativa dos valores de PA sistólica avaliados por medição em consulta. Atendendo a que os dados de eficácia da terapêutica na redução dos valores de PA medidos em consulta provêm exclusivamente dos dois ensaios realizados em adolescentes, coloca-se como hipótese que o eventual benefício da terapêutica hipouricemiante na diminuição da pressão arterial seja específico desta população mais jovem com pré-hipertensão / hipertensão arterial primária ligeira ou de instalação recente. Se considerarmos os dados de eficácia da terapêutica hipouricemiante na redução dos valores de PA obtidos na medição em ambulatório de 24 horas, apenas no subgrupo dos adolescentes, estes são concordantes com a existência de benefício nesta subpopulação. Em 2012, Soletsky e colaboradores investigaram se a hiperuricemia seria um mediador de hipertensão e não apenas um marcador bioquímico nesta subpopulação, através da inclusão de um grupo de doentes tratados com probenecid ( $n=19$ adolescentes) no seu ensaio. Este fármaco induz uricosúria através da inibição da absorção tubular de ácido úrico. Ao contrário de outros fármacos hipouricemiantes, não parece modular o stress oxidativo, uma vez que não exerce qualquer efeito na xantina-oxidase. O impacto na redução da pressão arterial foi semelhante no grupo tratado com alopurinol e no grupo tratado com probenecid, pelo que os autores sugeriram que o ácido úrico poderia desempenhar um papel na patogénese da hipertensão arterial. ${ }^{3}$ Estudos prévios em modelos animais sugeriram que o envolvimento do ácido úrico na patogénese da hipertensão arterial ocorre em duas fases - uma primeira modulável pelos agentes hipouricemiantes e uma segunda, mais tardia, refratária a estes agentes e associada a disfunção renal microvascular e inflamação tubulointersticial já estabelecidas. ${ }^{4}$ Do ponto de vista teórico, a conjugação destes dados permite especular que existirão diferenças fisiopatológicas na relação entre hiperuricemia e hipertensão arterial em populações mais jovens, comparativamente à população adulta, o que poderá contribuir para explicar a divergência de resultados quanto à eficácia da terapêutica hipouricemiante na redução da PA nestas duas populações. Este construto teórico assenta em resultados cuja evidência é de baixa qualidade, pelo que deve ser interpretado com cautela e encarado como uma hipótese que carece de investigação dirigida.

Os três ensaios incluídos na revisão sistemática, apesar de bem desenhados, apresentam algumas limitações, nomeadamente: amostra pequena (211 doentes); exclusão de hipertensão grave; e duração mínima de tratamento de apenas quatro semanas - o que limita a obtenção de resultados de eficácia e segurança a médio-longo prazo. Atendendo a que os três ensaios foram realizados em centros nos Estados Unidos da América, os resultados carecem de validação externa quanto à reprodutibilidade noutras populações. O reduzido número de doentes avaliados e a existência de heterogeneidade significativa para o objectivo primário poderá explicar o facto de os resultados não serem estatisticamente significativos mesmo perante a existência de um eventual benefício da terapêutica hipouricemiante na redução da PA.

Apesar de a suspensão da terapêutica devido a efeitos adversos não ter sido superior nos grupos submetidos a intervenção terapêutica, verificou-se que um doente medicado com alopurinol desenvolveu uma reação cutânea grave. A ocorrência de efeitos adversos associados ao alopurinol é incomum, mas potencialmente fatal, e ocorre mais frequentemente em doentes com doença renal crónica. ${ }^{5}$ Perante a qualidade muito baixa da evidência relativa à segurança, são necessários mais estudos para esclarecer o perfil de segurança desta intervenção nestes doentes.

Por fim, os autores identificaram três estudos que se encontravam a decorrer à data da revisão sistemática e que não foram incluídos na meta-análise. Os resultados de um destes estudos foram entretanto publicados 6 , tendo sido reportadas diminuições significativas na PA sistólica e diastólica em doentes hemodialisados. Neste sentido, revisões futuras desta revisão sistemática poderão apresentar conclusões diferentes. 


\section{IMPLICAÇÕES PARA A PRÁTICA CLÍNICA}

- A evidência disponível à data de realização da revisão sistemática é insuficiente para concluir se a utilização de terapêutica hipouricemiante é eficaz na redução da PA.

- A terapêutica hipouricemiante poderá ser eficaz na redução da PA na população específica dos adolescentes com pré-hipertensão ou hipertensão arterial ligeira

\section{REFERÊNCIAS}

1. Gois PH, Souza ER. Pharmacotherapy for hyperuricemia in hypertensive patients. Cochrane Database Syst Rev. 2017;4:CD008652.

2. Feig DI, Mazzali M, Kang DH, Nakagawa T, Price K, Kannelis J, et al. Serum uric acid: a risk factor and a target for treatment? J Am Soc Nephrol. 2006;17:S69-73.

3. Soletsky B, Feig DI. Uric acid reduction rectifies prehypertension in obese adolescents. Hypertension. 2012;60:1148-56.

4. Mazzali M, Kanellis J, Han L, Feng L, Xia YY, Chen Q, et al. Hyperuricemia induces a primary renal arteriolopathy in rats by a diagnosticada recentemente - hipótese que carece de validação em estudos futuros.

- A terapêutica hipouricemiante reduz eficazmente o valor sérico de ácido úrico.

- A qualidade da evidência existente é insuficiente para estabelecer a segurança da utilização da terapêutica hipouricemiante em doentes com pré-hipertensão ou hipertensão arterial primárias. blood pressure-independent mechanism. Am J Physiol Renal Physiol. 2002;282:F991-7.

5. Becker MA, Schumacher HR Jr, Wortmann RL, MacDonald PA, Eustace $D$, Palo WA, et al. Febuxostat compared with allopurinol in patients with hyperuricemia and gout. N Engl J Med. 2005;353:2450-61.

6. Omrani H, Sadeghi SM, Raeisi D, Hashemian A. The effect of allopurinol treatment regimen on serum uric acid and arterial blood pressure in hemodialysis patients. J Kermanshah Univ Med Sci. 2016;20:56-61.

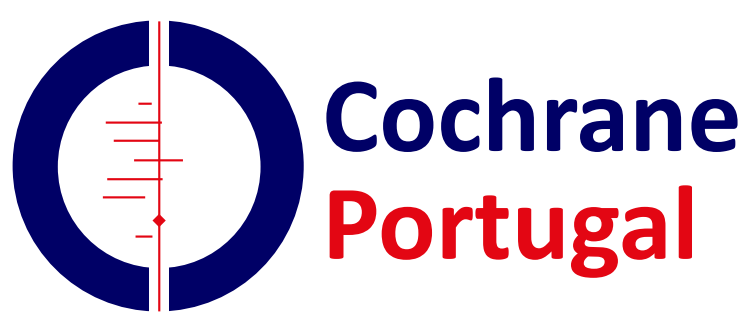

